Supporting Information for

\title{
Branching Corrected Mean Field Method for Nonadiabatic Dynamics
}

\author{
Jiabo Xu and Linjun Wang* \\ Center for Chemistry of Novel \& High-Performance Materials, and Department of \\ Chemistry, Zhejiang University, Hangzhou 310027, China \\ *Email: ljwang@zju.edu.cn
}

\section{Content}

1. BCMF-w Algorithm

2. Computational Details and Model Hamiltonians

3. Performance of EMF and BCMF in the Six Standard Models

4. Calculations with Strong Nuclear Quantum Effects in the Model Base 


\section{BCMF-w Algorithm}

Besides the algorithm based on stochastic wave function collapse, our branching corrected mean field (BCMF) method can also be realized by means of trajectory splitting. As an illustration, we consider a general system with one classical degree of freedom. However, the approach can be easily generalized to higher dimensions. The step-by-step algorithm of such BCMF based on weights (BCMF-w) is as follows:

(1) For each realization, we start with one mean field trajectory with weight $w=1$. The corresponding nuclear coordinate $\mathbf{R}$ and momentum $\mathbf{P}$ are sampled from the Wigner distribution of the initial nuclear wave function, $|\chi(\mathbf{R}, t=0)\rangle$.

(2) At each time step $t$, the momentum of wave packet (WP) on each potential energy surface (PES) $i\left(\boldsymbol{P}_{i}\right)$ is calculated through

$$
\begin{gathered}
\mathbf{P}_{i}=\eta \mathbf{P}, \\
\frac{\mathbf{P}_{i}^{2}}{2 m}+E_{i}=\frac{\mathbf{P}^{2}}{2 m}+E_{a v g},
\end{gathered}
$$

which are based on the parallel momenta and energy conservation approximations. Here, $\eta$ is a positive number, $m$ is the nuclear mass, $E_{i}$ and $E_{a v g}$ are energy of the $i$ th PES and the average PES at nuclear position $\mathbf{R}$. When the energy conservation cannot be fulfilled, $\mathbf{P}_{i}$ is assumed to be positive infinitesimal along the direction of $\mathbf{P}$.

(3) The nuclear coordinate and momentum at time $t+\Delta t$ are updated by solving the Newton equation,

$$
\left.\frac{\partial \mathbf{P}}{\partial t}=-\nabla_{\mathbf{R}}\langle\psi(\mathbf{r}, t)| \hat{H}_{0}(\mathbf{r}, \mathbf{R})\right)|\psi(\mathbf{r}, t)\rangle,
$$

where $\left.\hat{H}_{0}(\mathbf{r}, \mathbf{R})\right)$ is the electronic Hamiltonian at nuclear position $\mathbf{R}$. The electronic wave function is propagated through the time dependent Schrödinger equation, 


$$
i \hbar \frac{d c_{i}(t)}{d t}=\frac{-\mathbf{P} \cdot \mathbf{P}_{i}}{m} c_{i}-\sum_{j} i \hbar c_{j} \frac{\mathbf{P}}{m} \cdot \mathbf{d}_{i j}
$$

where $c_{i}(t)$ is the expansion coefficient of the electronic wave function in the adiabatic representation, and $\mathbf{d}_{i j}$ is the corresponding nonadiabatic coupling vector. In Eq. (S4), the effective Hamiltonian with phase correction has been utilized.

(4) For each PES $i$, we further calculate the nuclear momentum at time $t+\Delta t$ with $\mathbf{P}_{i}(t+\Delta t)=\mathbf{P}_{i}(t)+\mathbf{F}_{i}(t) \Delta t$, where $\mathbf{F}_{i}$ is the corresponding adiabatic Hellmann-Feynman force. If the dot products $\mathbf{F}_{i}(t) \cdot \mathbf{P}_{i}(t)$ and $\mathbf{F}_{i}(t) \cdot \mathbf{P}_{i}(t+\Delta t)$ have different signs, a WP reflection event is supposed to take place on the $i$ th PES during the time interval $\Delta t$. Similarly, for the average PES, the momentum at time $t+\Delta t$ is also calculated with $\mathbf{P}(t$ $+\Delta t)=\mathbf{P}(t)+\mathbf{F}_{a v g}(t) \Delta t$, where $\mathbf{F}_{\text {avg }}$ is the mean field force on the right-hand side of Eq. (S3). If $\mathbf{F}_{a v g}(t) \cdot \mathbf{P}(t)$ and $\mathbf{F}_{a v g}(t) \cdot \mathbf{P}(t+\Delta t)$ have different signs, the effective WP is supposed to be reflected on the average PES.

(5) When WP reflection happens, a single EMF trajectory cannot describe the dynamics of the whole system anymore. In the first two types of trajectory branching, some of the WPs on adiabatic PESs get reflected while the others do not. Here, we classify the quantum states into two subgroups: one with reflected WPs (group RG) and the other with nonreflected WPs (group NRG), whose populations are calculated as $P_{R G}=\sum_{i \in R G}\left|c_{i}\right|^{2}$ and $P_{N R G}=\sum_{i \in N R G}\left|c_{i}\right|^{2}$, respectively. Then, the parent trajectory is split into two child trajectories, which are explicitly propagated during the following dynamics in an independent way. For the reflected trajectory, we reset the wave function coefficients to $c_{i \in N R G}^{\prime}=0$ and $c_{i \in R G}^{\prime}=c_{i} / P_{R G}^{1 / 2} ;$ For the nonreflected 
trajectory, the coefficients are modified as $c_{i \in R G}^{\prime}=0$ and $c_{i \in N R G}^{\prime}=c_{i} / P_{N R G}^{1 / 2}$. For each new trajectory, the average PES is adjusted accordingly and the nuclear momentum is rescaled to conserve the total energy through

$$
\mathbf{P}^{\prime}(t)=\mathbf{P}(t) \sqrt{1+\frac{E_{a v g}-E_{a v g}^{\prime}}{\mathbf{P}(t)^{2} / 2 m}},
$$

where $E_{a v g}$ and $E_{a v g}^{\prime}$ are the averaged energies before and after resetting the wave function coefficients. The weights of the two child trajectories are updated as $w_{R G}=w_{p} P_{R G}$ and $w_{N R G}=w_{p} P_{N R G}$, where $w_{p}$ is the weight of the parent trajectory. If the energy conservation is violated, the trajectory spliting is cancelled. However, it is possible that a WP satisfies the energy conservation at $t-\Delta t$ and gets reflected during $[t-\Delta t, t]$, but the energy conservation does not hold at time $t$. In this special case, the WP branching is still accepted, and the new momentum of the corresponding child trajectory is simply reset to zero. In the third type of trajectory branching, the WPs on all adiabatic PESs are not reflected while the effective WP gets reflected. In this case, trajectory splitting will not take place as all the WPs on adiabatic PESs still move in a similar manner. Here, we need to make special corrections to the parent trajectory. The trajectory will switch to a new effective PES constructed only by energy-allowed states. Namely, the electronic states are classified into two groups: the energy-forbidden group (EFG) with potential energies larger than the total energy and the energy-allowed group (EAG) with potential energies smaller than the total energy. Then, the electronic wave function coefficients are reset to

$$
c_{i \in E F G}^{\prime}=0 \text {, }
$$




$$
c_{i \in E A G}^{\prime}=c_{i} / \sqrt{\sum_{j \in E A G}\left|c_{j}\right|^{2}}
$$

and the nuclear momentum is adjusted by Eq. (S5) to conserve the total energy.

(6) Repeat steps (2)-(5) until a predefined criterion is satisfied. During this process, new child trajectories are generated until the total number of trajectories in the corresponding realization reaches a preset parameter $N_{b}$. After then, when additional trajectory branching happens, the parent trajectory adopts the reflected or nonreflected configuration with larger population while retaining the same weight. Besides, all trajectories are propagated independently according to Eqs. (S3) and (S4).

(7) To properly describe the asymptotic region, the electronic coefficients of each trajectory are transformed according to Eqs. (S6) and (S7) with energy conservation taken into account. Finally, the electronic population of the $i$ th state is calculated by $P_{i}=\sum_{j k} w_{j k}\left|c_{i j k}^{\prime}\right|^{2}$, where $c_{i j k}^{\prime}$ is the transformed electronic coefficient of $k$ th branched trajectory in the $j$ th realization and $w_{j k}$ is the corresponding weight. 


\section{Computational Details and Model Hamiltonians}

In all nonadiabatic dynamics simulations of one-dimensional two-level systems, a Gaussian WP is placed on the negative $x$ side of the lower adiabatic PES with a positive momentum a $t=0$. The nuclear wave function is expressed as

$$
\chi(x, 0)=\left(\frac{1}{2 \pi \sigma_{x}^{2}}\right)^{1 / 4} \exp \left[-\frac{\left(x-x_{0}\right)^{2}}{4 \sigma_{x}^{2}}+i k\left(x-x_{0}\right)\right] .
$$

Here, $\sigma_{x}$ is the spatial with of the WP, $x_{0}$ and $k$ refer to the central position and momentum of the WP, respectively. In the present study, we set $x_{0}=x_{l e f t}-3 \sigma_{x}$, where $x_{\text {left }}$ indicates the left border of the nonadiabatic coupling region and varies in different models. In mean field calculations, the initial coordinates and momenta of different realizations are sampled by the corresponding Wigner distribution,

$$
P(x, p)=\frac{1}{\sqrt{2 \pi} \sigma_{x}} \exp \left[-\frac{\left(x-x_{0}\right)^{2}}{2 \sigma_{x}^{2}}\right] \cdot \frac{1}{\sqrt{2 \pi} \sigma_{p}} \exp \left[-\frac{\left(p-p_{0}\right)^{2}}{2 \sigma_{p}^{2}}\right],
$$

where $p_{0}=\hbar k$ and $\sigma_{p}=\hbar /\left(2 \sigma_{x}\right)$. A large number of trajectories are utilized in BCMF-s calculations to reduce the errors caused by the initial sampling of coordinates and momenta and stochastic collapse of the electronic wave function. The exact quantum dynamics are simulated by the discrete variable representation (DVR) method with transmission-free absorbing potentials. ${ }^{1-3}$ In all investigated systems, the mass of the particle is always set as 2000 a.u. Atomic units are used unless otherwise noted.

We first consider six widely studied standard scattering models, which are the simple avoided crossing (SAC), the dual avoided crossing (DAC), the extended coupling with reflection (ECR), the dumbbell geometry (DBG), the double arch geometry (DAG), and the dual Rosen-Zener-Demkov noncrossing (DRN) models. ${ }^{4-6}$ 
Different $x_{\text {left }}$ and $\sigma_{x}$ are considered. In SAC, DAC and ECR models, we set $x_{\text {left }}=-17.5$ and $\sigma_{x}=10 / k$; In the DBG model, $x_{\text {left }}=-22.5$ and $\sigma_{x}=3 \sqrt{2} / 2 ;$ In the DAG model, $x_{l e f t}=-27.5$ and $\sigma_{x}=2.0$; in the DRN model, $x_{l e f t}=-12.5$ and $\sigma_{x}=0.5$. In mean field calculations, the time step size is set as $d t=0.1 .50000$ trajectories are carried out in each BCMF-s calculation of these models.

The diabatic electronic Hamiltonians for the six models are provided one by one below. For the SAC model, the Hamiltonian elements are given by

$$
\begin{gathered}
H_{11}(x)=\left\{\begin{array}{l}
-A[1-\exp (B x)], x<0 \\
A[1-\exp (-B x)], x>0
\end{array}\right. \\
H_{22}(x)=-H_{11}(x), \\
H_{12}(x)=H_{21}(x)=C \exp \left(-D x^{2}\right),
\end{gathered}
$$

where $A=0.01, B=1.6, C=0.005$, and $D=1$. The DAC Hamiltonian reads

$$
\begin{gathered}
H_{11}(x)=0, \\
H_{22}(x)=E_{0}-A \exp \left(-B x^{2}\right), \\
H_{12}(x)=H_{21}(x)=C \exp \left(-D x^{2}\right),
\end{gathered}
$$

with $E_{0}=0.05, A=0.1, B=0.28, C=0.015$, and $D=0.06$. In the ECR model, the Hamiltonian is defined as

$$
\begin{gathered}
H_{11}(x)=A, \\
H_{22}(x)=-A, \\
H_{12}(x)=H_{21}(x)=\left\{\begin{array}{l}
B \exp (C x), x<0 \\
B[2-\exp (-C x)], \quad x>0
\end{array}\right.
\end{gathered}
$$

where $A=6 \times 10^{-4}, B=0.1$, and $C=0.9$. The DBG model is a symmetrized combination of two ECR models. The Hamiltonian is given by 


$$
\begin{gathered}
H_{11}(x)=A, \\
H_{22}(x)=-A, \\
H_{12}(x)=H_{21}(x)=\left\{\begin{array}{l}
B \exp [C(x-Z)]+B\{2-\exp [C(x+Z)]\}, \quad x<-Z \\
B \exp [C(x-Z)]+B \exp [-C(x+Z)],-Z<x<Z \\
B \exp [-C(x+Z)]+B\{2-\exp [-C(x-Z)]\}, \quad x>Z
\end{array}\right.
\end{gathered}
$$

where $A=6 \times 10^{-4}, B=0.1, C=0.9$, and $Z=10$. Similarly, the DAG model is defined based on two ECR models,

$$
\begin{gathered}
H_{11}(x)=A, \\
H_{22}(x)=-A, \\
H_{12}(x)=H_{21}(x)=\left\{\begin{array}{l}
-B \exp [C(x-Z)]+B \exp [C(x+Z)], \quad x<-Z \\
-B \exp [C(x-Z)]-B \exp [-C(x+Z)]+2 B, \quad-Z<x<Z \\
B \exp [-C(x-Z)]-B \exp [-C(x+Z)], \quad x>Z
\end{array}\right.
\end{gathered}
$$

where $A=6 \times 10^{-4}, B=0.1, C=0.9$, and $Z=4$. The sixth model is the DRN model with

$$
\begin{gathered}
H_{11}(x)=0, \\
H_{22}(x)=E_{0}, \\
H_{12}(x)=H_{21}(x)=A\left\{\exp \left[-B(x-Z)^{2}\right]+\exp \left[-B(x+Z)^{2}\right]\right\},
\end{gathered}
$$

where $E_{0}=0.01, A=0.03, B=3.2$, and $Z=2$.

A carefully designed one-dimensional two-level model base is utilized to further benchmark the robustness of mean field algorithms. ${ }^{7}$ Five different expressions are considered for the diagonal elements of the diabatic Hamiltonian, which are

$$
\begin{gathered}
H_{11}^{[1]}=E_{1}, \\
H_{22}^{[1]}=E_{2}, \\
H_{11}^{[2]}= \begin{cases}E_{2}-A_{1}\left(1-\exp \left(B_{1} x\right)\right), & x<0 \\
E_{2}+A_{1}\left(1-\exp \left(-B_{1} x\right)\right), & x>0\end{cases}
\end{gathered}
$$




$$
\begin{aligned}
H_{22}^{[2]}= \begin{cases}E_{3}-A_{1}\left(1-\exp \left(B_{1} x\right)\right), & x<0 \\
E_{3}+A_{1}\left(1-\exp \left(-B_{1} x\right)\right), & x>0\end{cases} \\
H_{11}^{[3]}= \begin{cases}-E_{2}+A_{1}\left(1-\exp \left(B_{1} x\right)\right), & x<0 \\
-E_{2}-A_{1}\left(1-\exp \left(-B_{1} x\right)\right), & x>0\end{cases} \\
H_{22}^{[3]}= \begin{cases}E_{1}+A_{1}\left(1-\exp \left(B_{1} x\right)\right), & x<0 \\
E_{1}-A_{1}\left(1-\exp \left(-B_{1} x\right)\right), & x>0\end{cases} \\
H_{11}^{[4]}=E_{1}+A_{2} \exp \left(-B_{2} x^{2}\right), \\
H_{22}^{[4]}=E_{2}+A_{2} \exp \left(-B_{2} x^{2}\right), \\
H_{11}^{[5]}=E_{1}-A_{2} \exp \left(-B_{2} x^{2}\right),
\end{aligned}
$$

where $A_{1}=0.01, B_{1}=1.6, A_{2}=0.02, B_{2}=0.28, E_{1}=0, E_{2}=0.01$, and $E_{3}=0.02$. For the off-diagonal terms of the Hamiltonian, we consider a Gaussian function

$$
H_{12}=H_{21}=A_{3} \exp \left(-B_{3} x^{2}\right),
$$

where $A_{3}$ and $B_{3}$ characterize the strength and width of the diabatic coupling, respectively. Four different $A_{3}$ (i.e., $0.0025,0.005,0.02$, and 0.04 ) and two different $B_{3}$ (i.e., 0.4 and 1.5) are considered in the present study. There exist 25 and 8 combinations for the diagonal and off-diagonal terms of the diabatic Hamiltonian, respectively. Thereby, a total number of 200 models are constructed in our model base. For simplicity, we use $M_{i j}^{A_{3}, B_{3}}$ to express the model using $H_{11}^{[i]}, H_{22}^{[j]}$, and $H_{12}$ with parameters $A_{3}$ and $B_{3}$ to construct the Hamiltonian. For all models in the model base, we choose $x_{\text {left }}$ $=-12.5$ and $\sigma_{x}=10 / k$. In mean field calculations, $d t=0.05$ is generally utilized, and a smaller $d t$ in the range of 0.0005-0.01 is used for models suffering from severe trivial crossing problems. ${ }^{8-13} 10000$ trajectories are considered in each BCMF-s calculation. 


\section{Performance of EMF and BCMF in the Six Standard Models}

In Fig. S1, we show the adiabatic potential energy surfaces and the corresponding nonadiabatic couplings of the six standard models. As shown in Fig. S2-S7, the results of the traditional Ehrenfest mean field (EMF) and our BCMF are compared with the exact quantum solutions by DVR. ${ }^{1-3}$ For each model, the populations of transmission and reflection on the lower and upper surfaces are shown as a function of the initial nuclear momentum. In all the investigated channels, the results of our BCMF method are almost on top of the exact quantum solutions by DVR.
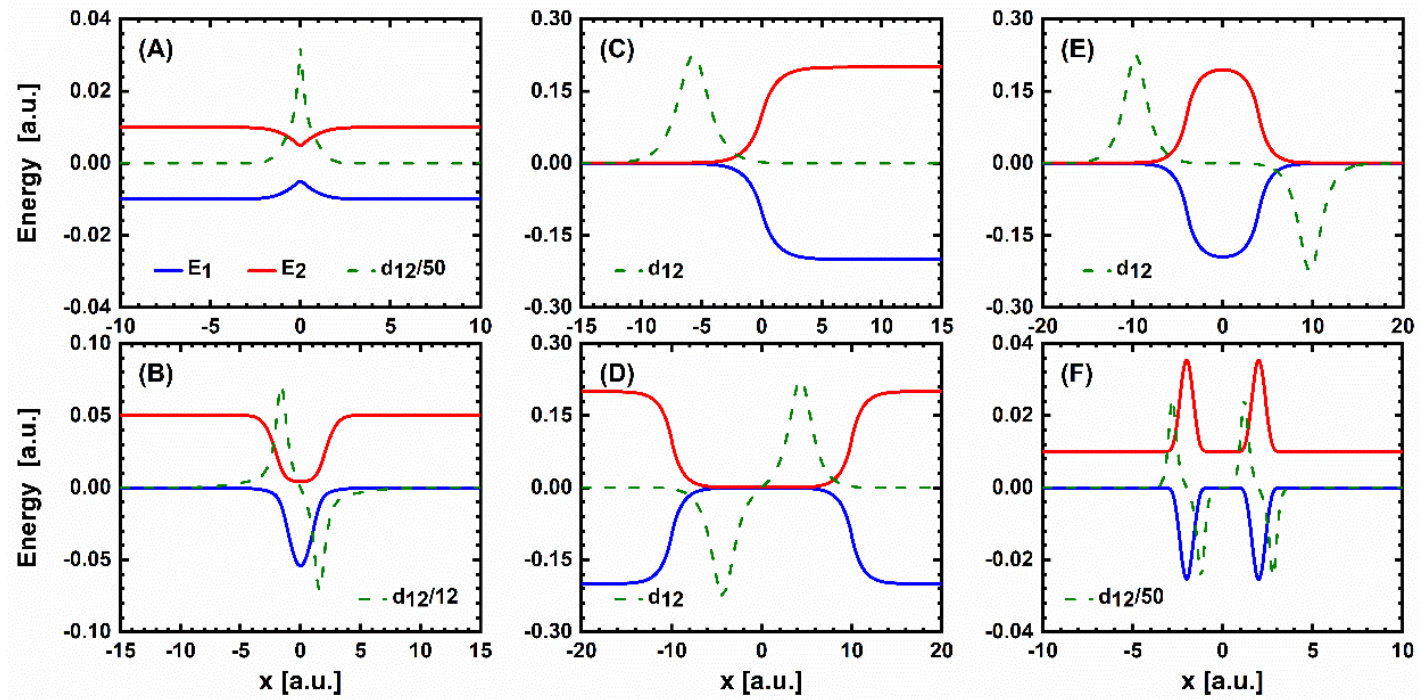

FIGURE S1. Adiabatic potential energy surfaces (red and blue solid lines) and the nonadiabatic couplings (green dashed lines) of (A) SAC, (B) DAC, (C) ECR, (D) DBG, (E) DAG, and (F) DRN models. 

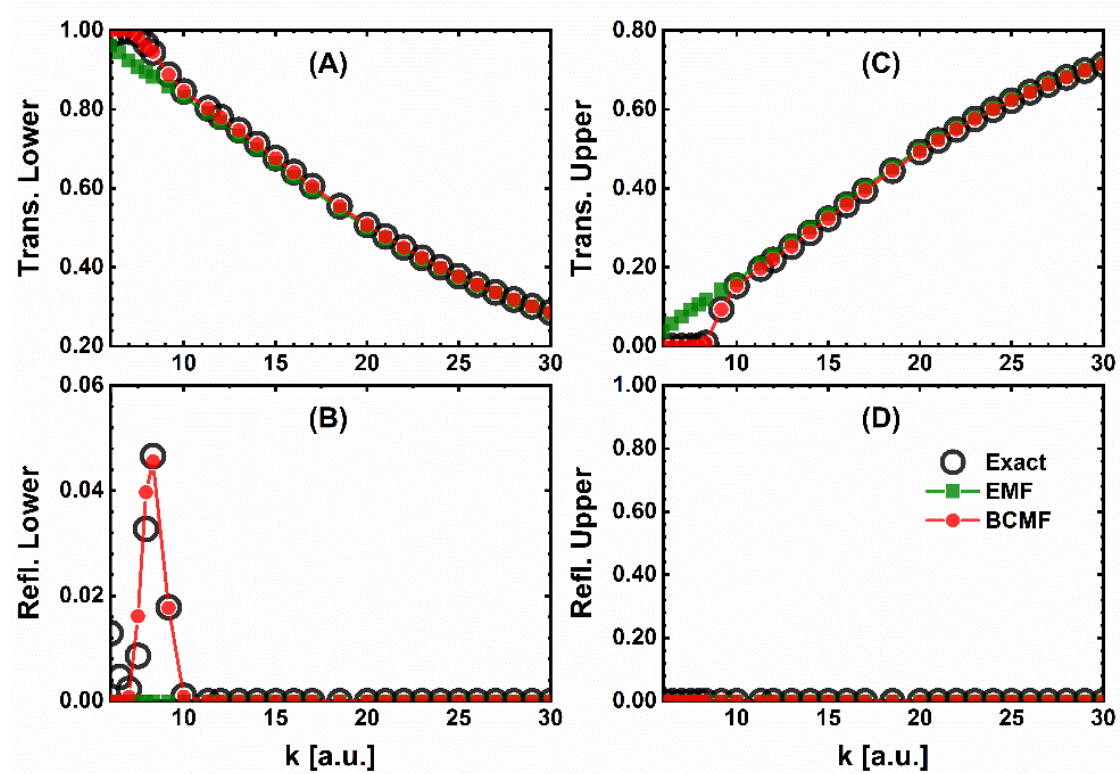

FIGURE S2. (A) Transmission and (B) reflection populations on the lower surface, (C) transmission and (D) reflection populations on the upper surface for the SAC model. Open circles are exact quantum solutions by DVR. The results of EMF and BCMF are shown as green solid squares and red solid circles, respectively.
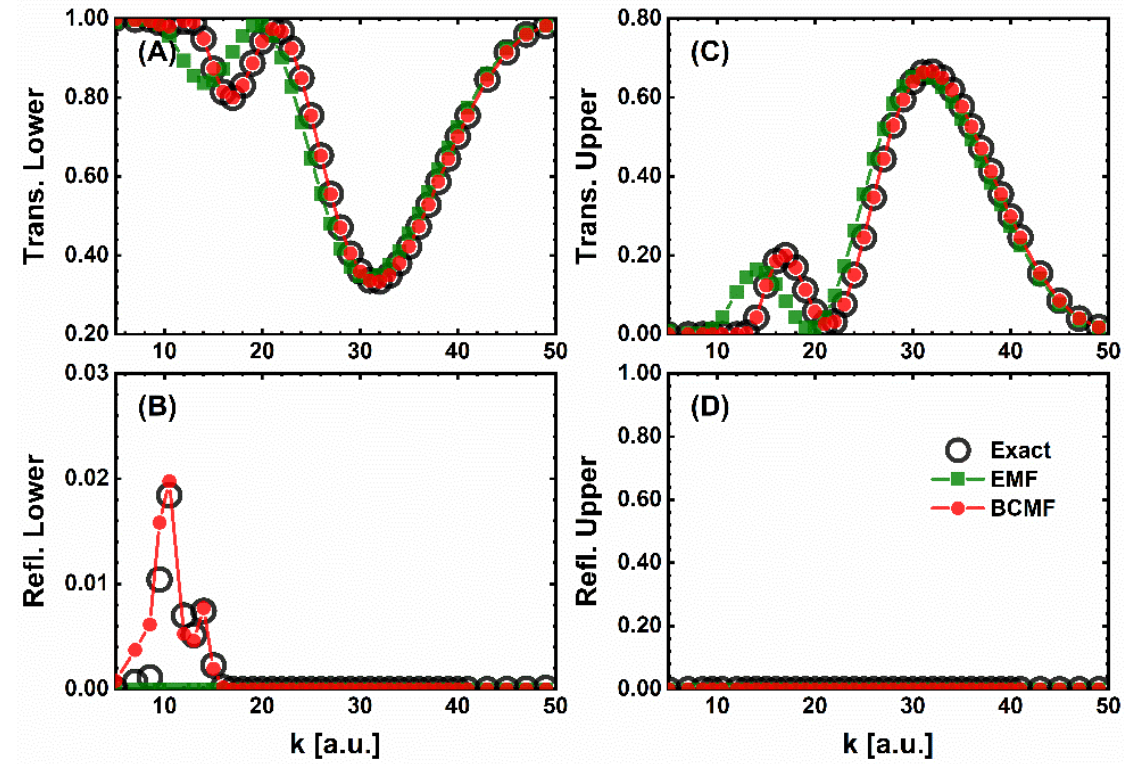

FIGURE S3. (A) Transmission and (B) reflection populations on the lower surface, (C) transmission and (D) reflection populations on the upper surface for the DAC model. The symbols are the same as those in Fig. S2. 

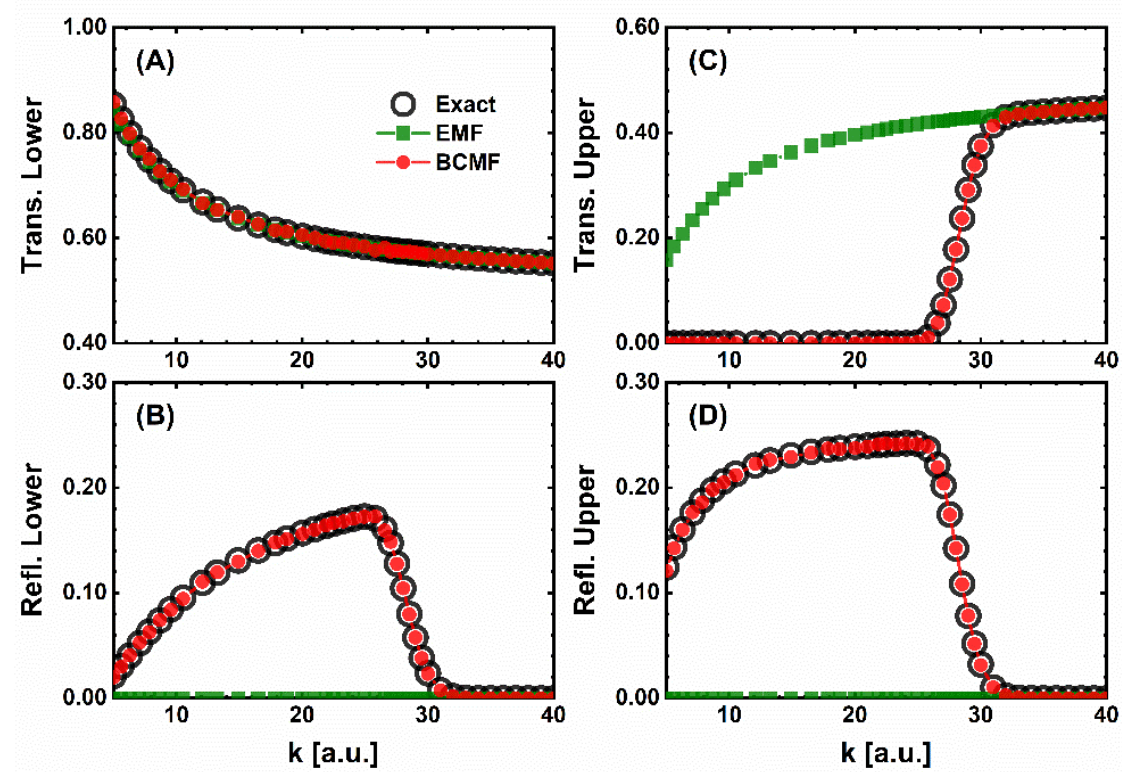

FIGURE S4. (A) Transmission and (B) reflection populations on the lower surface, (C) transmission and (D) reflection populations on the upper surface for the ECR model. The symbols are the same as those in Fig. S2.
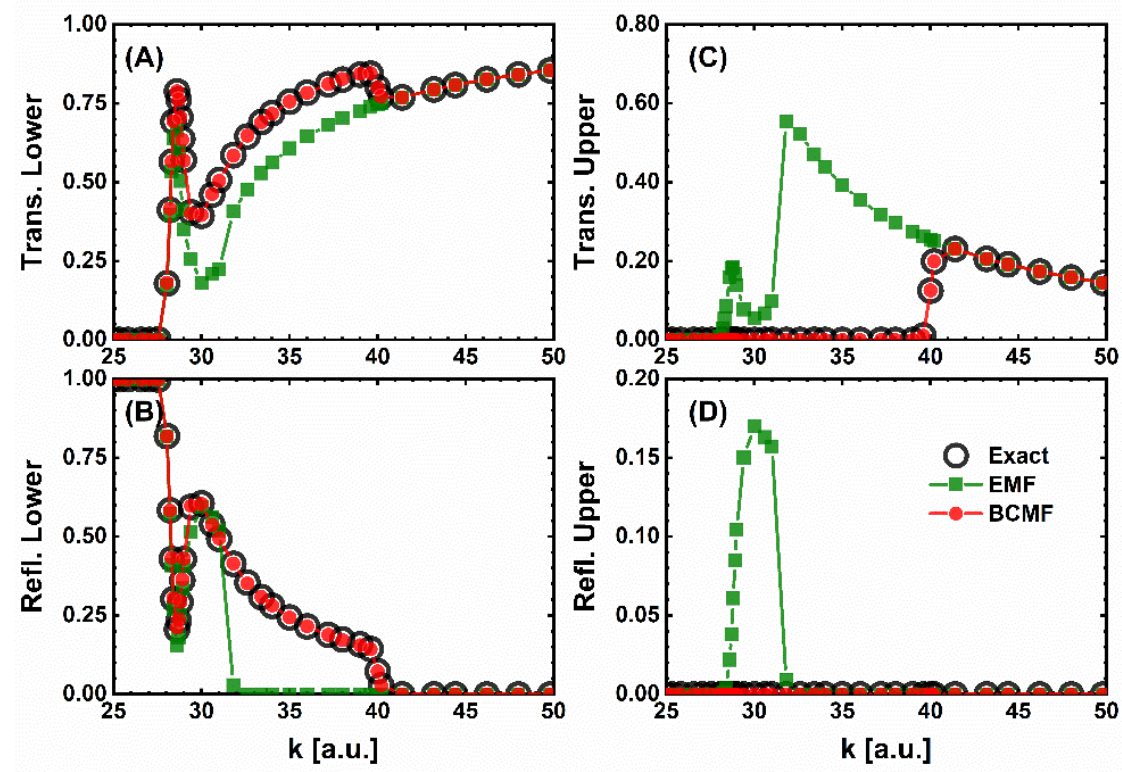

FIGURE S5. (A) Transmission and (B) reflection populations on the lower surface, (C) transmission and (D) reflection populations on the upper surface for the DBG model. The symbols are the same as those in Fig. S2. 

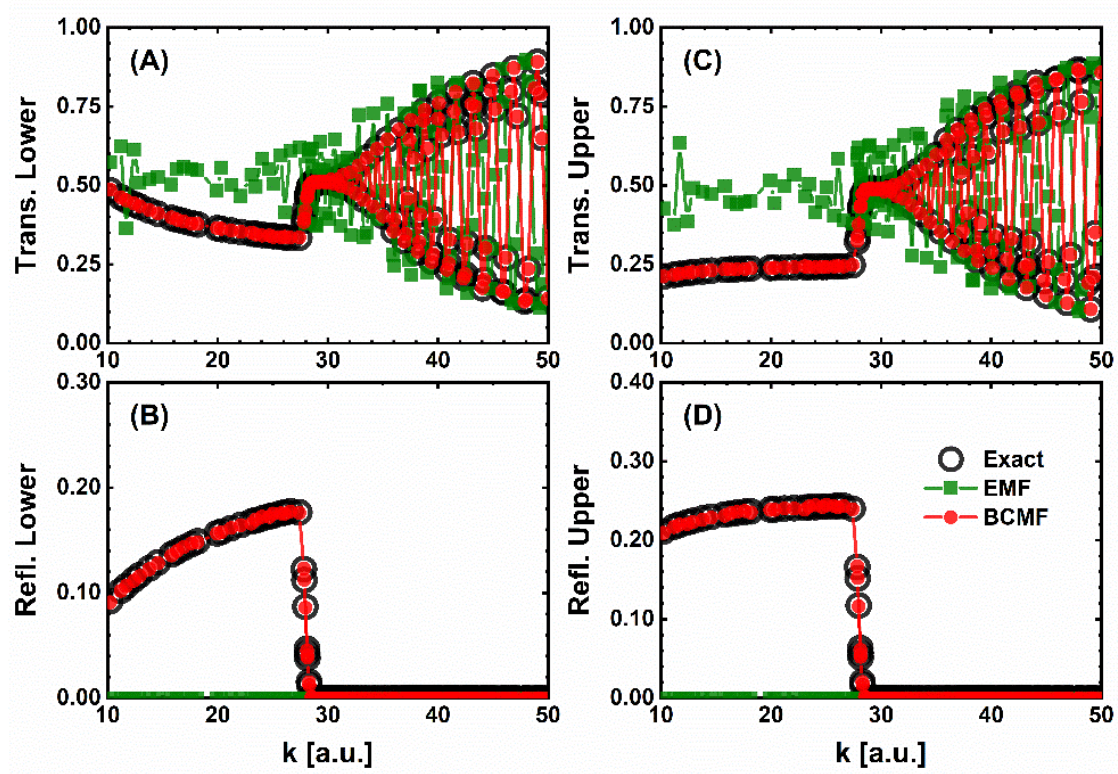

FIGURE S6. (A) Transmission and (B) reflection populations on the lower surface, (C) transmission and (D) reflection populations on the upper surface for the DAG model. The symbols are the same as those in Fig. S2.
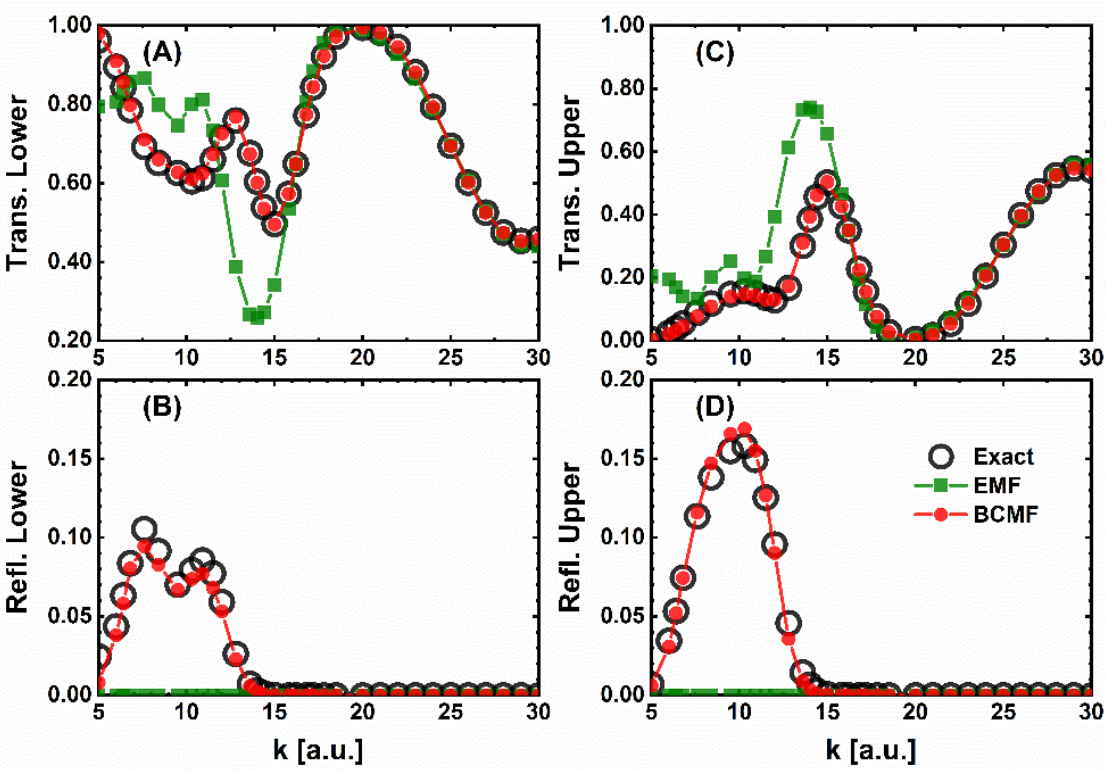

FIGURE S7. (A) Transmission and (B) reflection populations on the lower surface, (C) transmission and (D) reflection populations on the upper surface for the DRN model. The symbols are the same as those in Fig. S2. 


\section{Calculations with Strong Nuclear Quantum Effects in the Model Base}

The diabatic potential energy surfaces, $H_{11}^{[i]}$ and $H_{22}^{[j]}$, in the model base are illustrated in Fig. S8. In our model base, there exist several calculations associated with strong nuclear quantum effects, which cannot be properly described by the classical Wigner approximation and result in relatively large simulation errors. We find that they share similar features: (1) at least one potential energy barrier with relatively narrow spatial width is present on the lower surface, and (2) the energy of one such potential barrier is very close to the total energy of the system. In Fig. $4 \mathrm{~B}$ of the main text, we have highlighted 10 population channels with relatively large errors in red. They belong to five calculations, whose PESs and the corresponding transmission and reflections results on both lower and upper surfaces are shown in Figs. S9-S13.

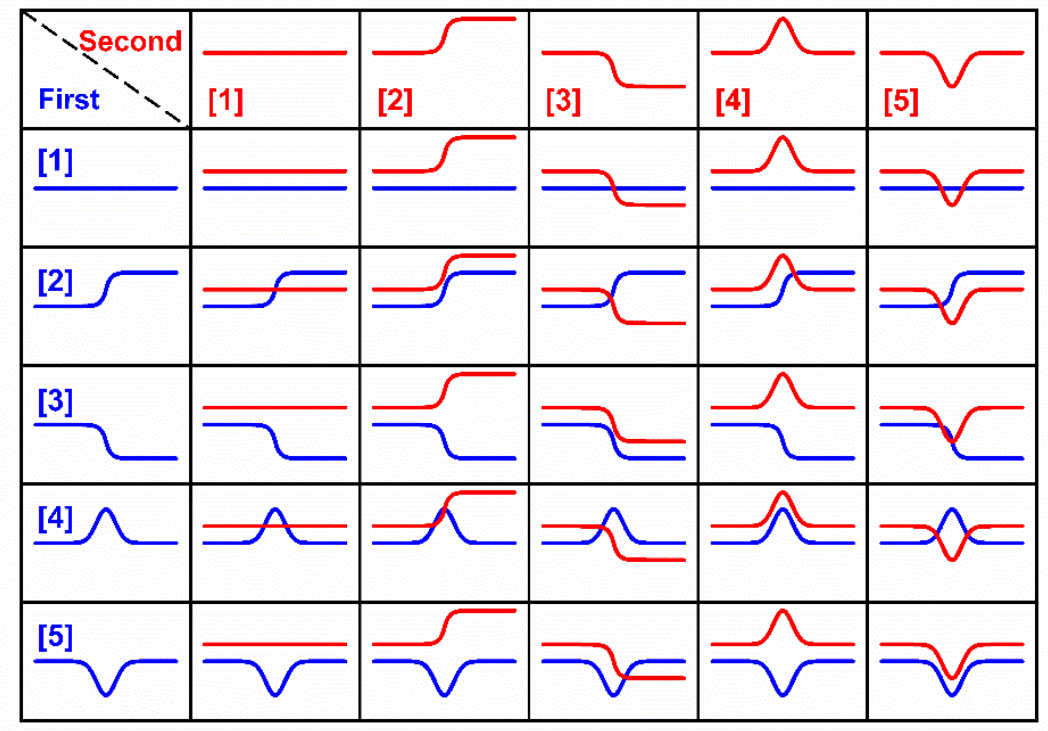

FIGURE S8. Diabatic potential energy surfaces to construct the one-dimensional twolevel model base. The first diabatic potentials $\left(H_{11}^{[i]}\right)$ are shown in blue while the second diabatic potentials $\left(H_{22}^{[j]}\right)$ are shown in red. The types of expression for the diagonal terms of the Hamiltonians are indicated by [1]-[5]. 

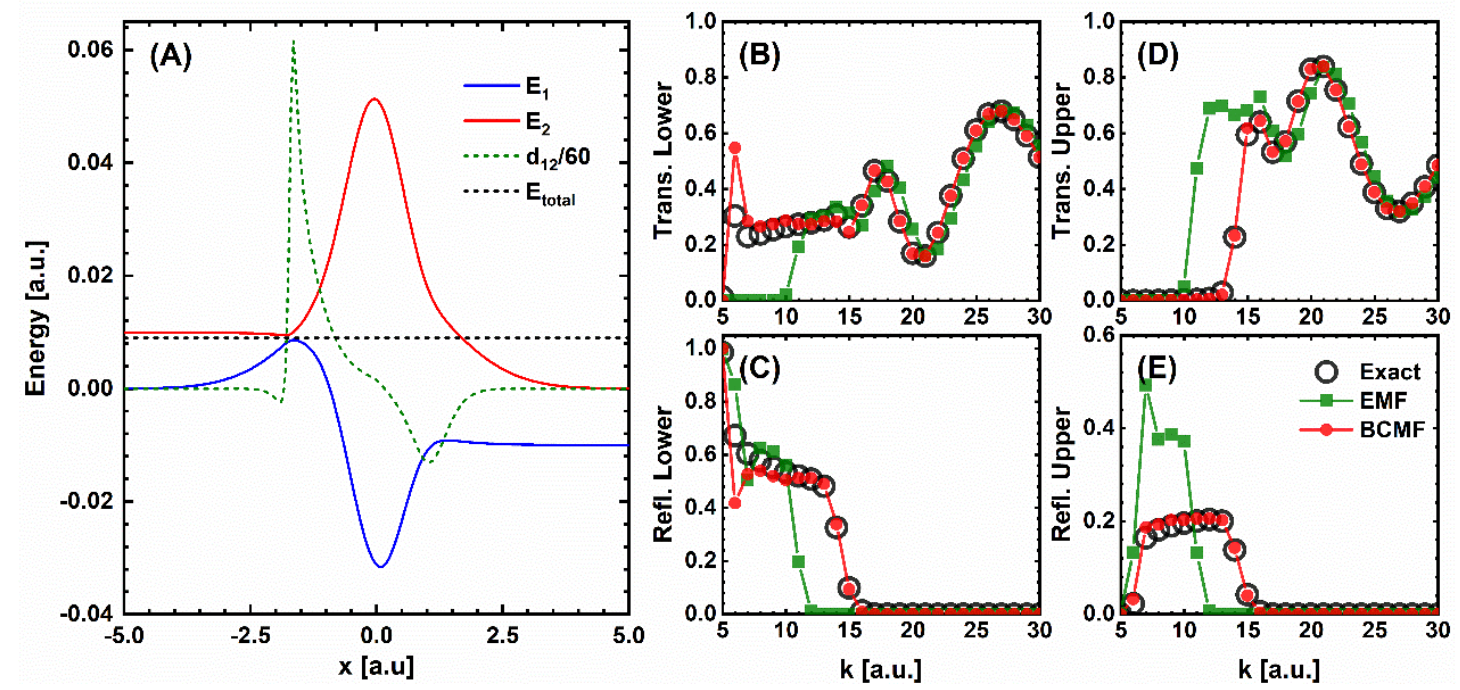

FIGURE S9. (A) Adiabatic PESs of the model $M_{43}^{0.04,1.5}$. The lower and upper adiabatic PESs are shown by red and blue solid lines, respectively. The nonadiabatic coupling is shown by the green dashed line, and the system energy is represented by the black dashed line with $k=6$. (B) Transmission and (C) reflection populations on the lower surface, (D) transmission and (E) reflection populations on the upper surface. The symbols in (B)-(E) are the same as those in Fig. S2. Relatively large errors arise on the transmission and reflection channels on the lower surface for $k=6$. 

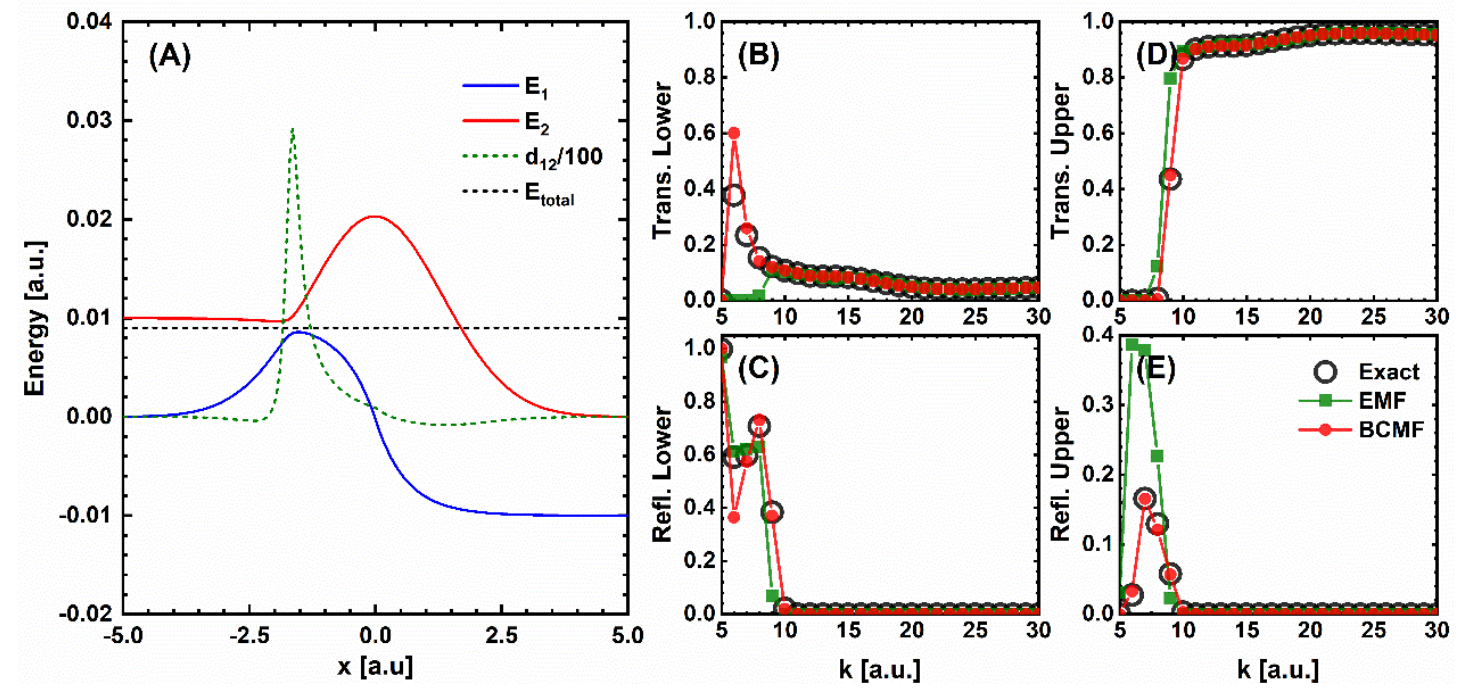

FIGURE S10. (A) Adiabatic PESs of the model $M_{43}^{0.0025,0.4}$, (B) transmission and (C) reflection populations on the lower surface, (D) transmission and (E) reflection populations on the upper surface. The lines and symbols are the same as those in Fig. S9. The black dashed line in (A) corresponds to the system energy with $k=6$. Relatively large errors arise on the transmission and reflection channels on the lower surface for $k$ $=6$. 

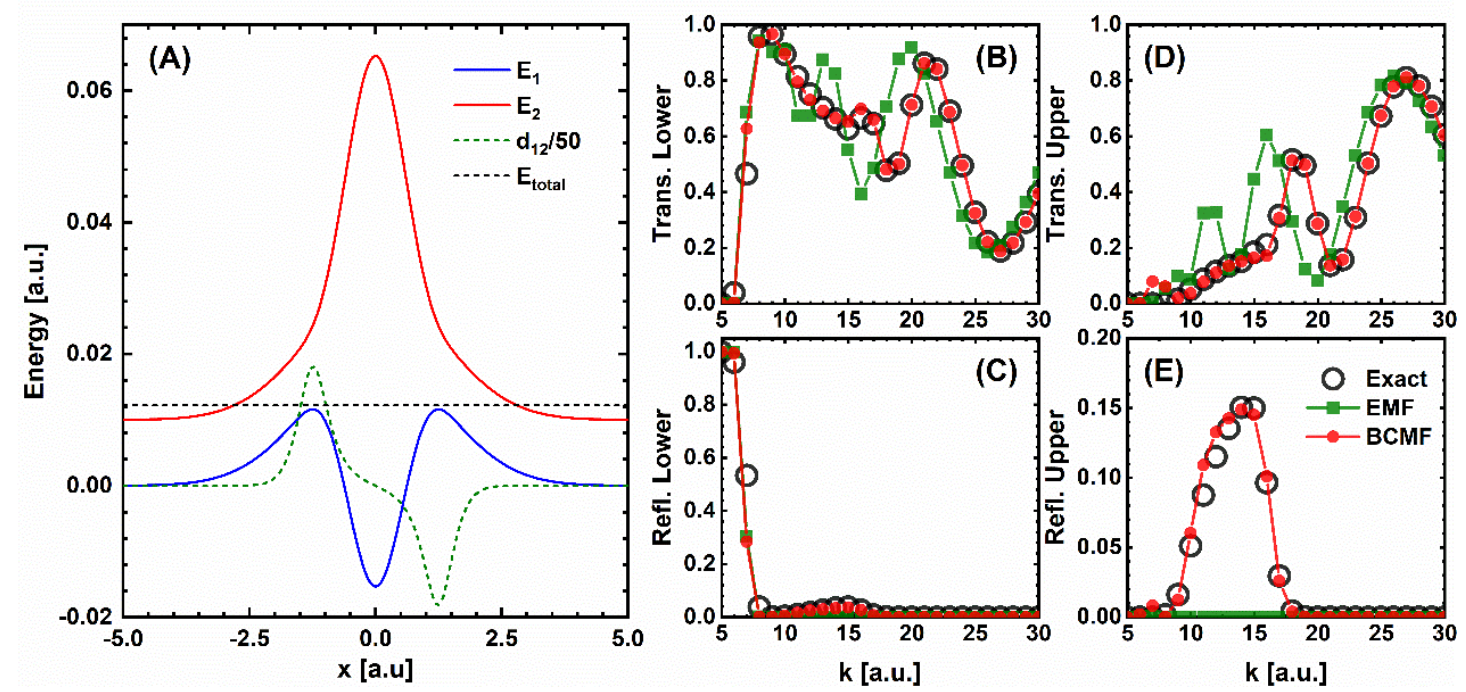

FIGURE S11. (A) Adiabatic PESs of the model $M_{44}^{0.04,1.5}$, (B) transmission and (C) reflection populations on the lower surface, (D) transmission and (E) reflection populations on the upper surface. The lines and symbols are the same as those in Fig. S9. The black dashed line in (A) corresponds to the system energy with $k=7$. Relatively large errors arise on the transmission and reflection channels on the lower surface for $k$ $=7$. 

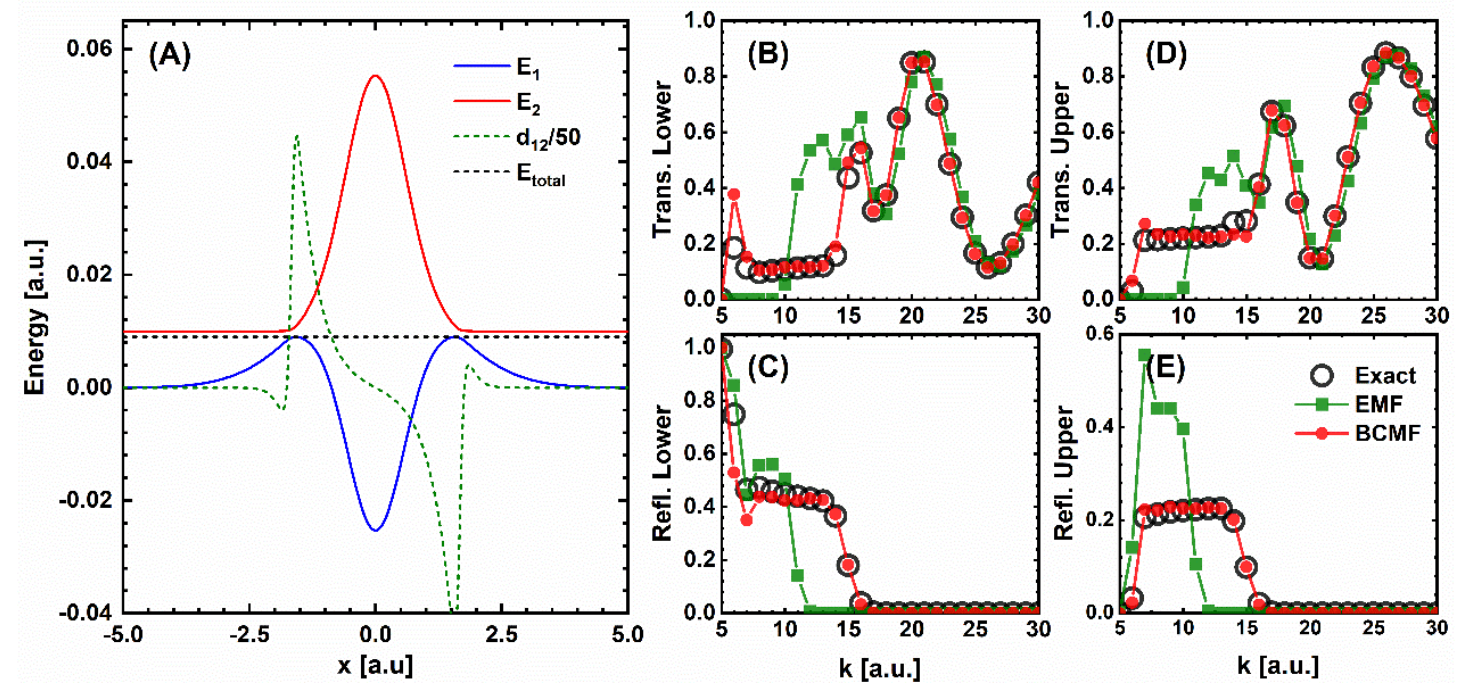

FIGURE S12. (A) Adiabatic PESs of the model $M_{41}^{0.04,1.5}$, (B) transmission and (C) reflection populations on the lower surface, (D) transmission and (E) reflection populations on the upper surface. The lines and symbols are the same as those in Fig. S9. The black dashed line in (A) corresponds to the system energy with $k=6$. Relatively large errors arise on the transmission and reflection channels on the lower surface for $k$ $=6$. 

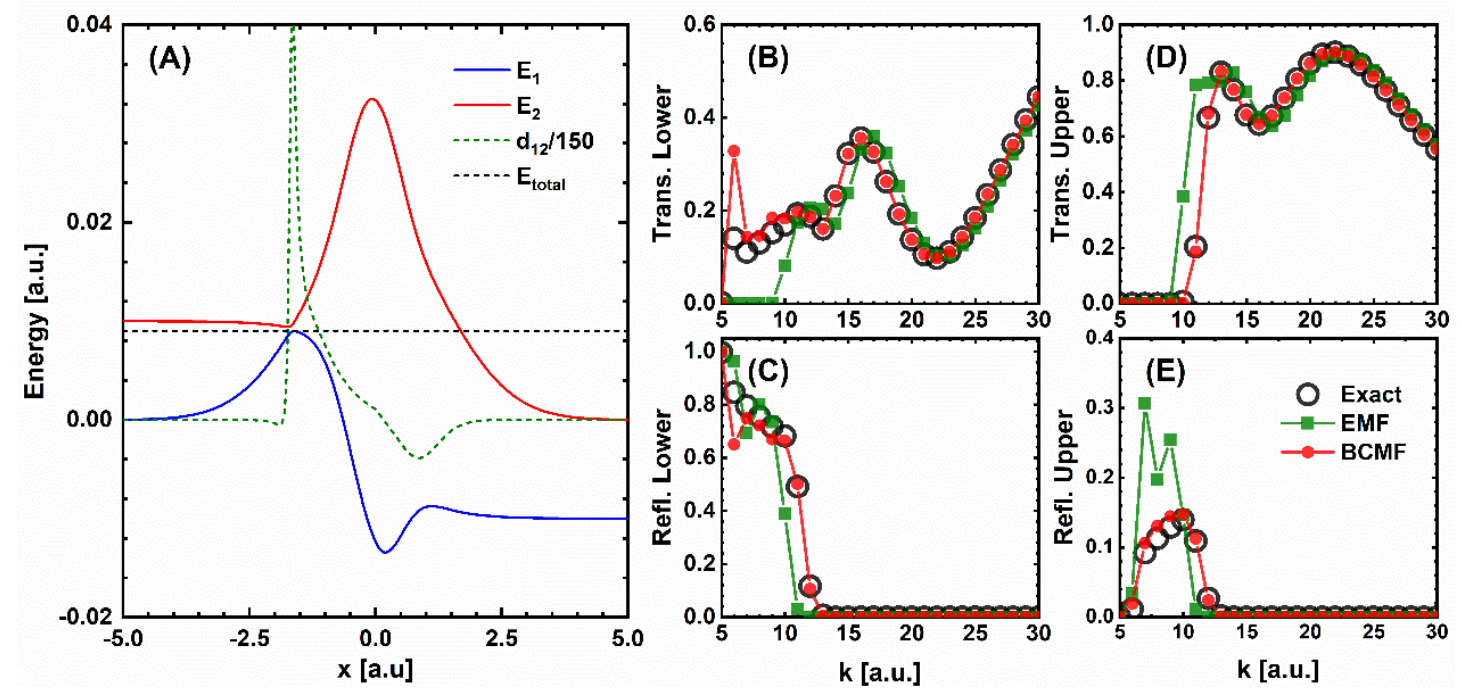

FIGURE S13. (A) Adiabatic PESs of the model $M_{43}^{0.02,1.5}$, (B) transmission and (C) reflection populations on the lower surface, (D) transmission and (E) reflection populations on the upper surface. The lines and symbols are the same as those in Fig. S9. The black dashed line in (A) corresponds to the system energy with $k=6$. Relatively large errors arise on the transmission and reflection channels on the lower surface for $k$ $=6$. 


\section{REFERENCES}

(1) Colbert, D. T.; Miller, W. H. A Novel Discrete Variable Representation for Quantum Mechanical Reactive Scattering via the $S$-matrix Kohn Method. J. Chem. Phys. 1992, 96, 1982-1991.

(2) Manolopoulos, D. E. Derivation and Reflection Properties of a Transmission-Free Absorbing Potential. J. Chem. Phys. 2002, 117, 9552-9559.

(3) Gonzalez-Lezana, T.; Rackham, E. J.; Manolopoulos, D. E. Quantum Reactive Scattering with a Transmission-Free Absorbing Potential. J. Chem. Phys. 2004, 120, 2247-2254.

(4) Tully, J. C. Molecular Dynamics with Electronic Transitions. J. Chem. Phys. 1990, 93, 1061-1071.

(5) Subotnik, J. E.; Shenvi, N. A New Approach to Decoherence and Momentum Rescaling in the Surface Hopping Algorithm. J. Chem. Phys. 2011, 134, 024105.

(6) Zhu, C. Restoring Electronic Coherence/Decoherence for a Trajectory-Based Nonadiabatic Molecular Dynamics. Sci. Rep. 2016, 6, 24198.

(7) Xu, J.; Wang. L. Branching Corrected Surface Hopping: Resetting Wavefunction Coefficients Based on Judgement of Wave Packet Reflection. J. Chem. Phys. 2019, 150, 164101.

(8) Granucci, G.; Persico, M.; Toniolo, A. Direct Semiclassical Simulation of Photochemical Processes with Semiempirical Wave Functions. J. Chem. Phys. 2001, $114,10608-10615$.

(9) Fernandez-Alberti, S.; Roitberg, A. E.; Nelson, T.; Tretiak, S. Identification of Unavoided Crossings in Nonadiabatic Photoexcited Dynamics Involving Multiple Electronic States in Polyatomic Conjugated Molecules. J. Chem. Phys. 2012, 137, 014512.

(10) Wang, L.; Prezhdo, O. V. A Simple Solution to the Trivial Crossing Problem in Surface Hopping. J. Phys. Chem. Lett. 2014, 5, 713-719.

(11) Bai, X.; Qiu, J.; Wang, L. An Efficient Solution to the Decoherence Enhanced Trivial Crossing Problem in Surface Hopping. J. Chem. Phys. 2018, 148, 104106.

(12) Qiu, J.; Bai, X.; Wang, L. Crossing Classified and Corrected Fewest Switches Surface Hopping. J. Phys. Chem. Lett. 2018, 9, 4319-4325.

(13) Qiu, J.; Bai, X.; Wang, L. Subspace Surface Hopping with Size-Independent Dynamics. J. Phys. Chem. Lett. 2019, 10, 637-644. 